\title{
Langzeitverhalten von weichballistischen Materialien
}

\author{
Anja Gäbler, Michael Hendrix, Michael Herzog, Eberhard Geßner
}

Zusammenfassung

Dieser Aufsatz befasst sich mit dem Langzeitverhalten ballistischer Materialien. Vorrangig soll die Frage geklärt werden, ob ballistisches Material qualitätsmindernde Erscheinungen im Schutzverhalten aufweist, die mit dem fortschreitenden Alter des Materials einhergehen. Um diese Frage zu klären, wurden anhand zahlreicher Beschussversuche an polizeilichen Schutzwesten statistisch verlässliche Daten gesammelt und anschließend unter verschiedenen Blickwinkeln ausgewertet. Zusätzlich wurden verschiedene Laborversuche am Aramid-Gewebe durchgeführt.

\section{Abstract}

This publication deals with the long-term behavior of ballistic material. It aims to answer the question, whether ballistic material shows significant signs of a deterioration that comes with preceding age. To answer that question numerous shooting tests with bulletproof vests were made to collect statistically reliable Data. The data was then evaluated under different point of views. Additionally various laboratory experiments were performed with Aramid fibre.

\section{Einführung}

Schutzsysteme aus weichballistischen Materialien spielen in vielen Bereichen aufgrund ihres geringen Gewichts bei hohem Schutz eine zunehmende Rolle. Ein bekanntes Beispiel eines ballistischen Schutzsystems sind polizeiliche Schutzwesten. Weichballistische Materialien spielen aber nicht nur als wesentlicher Bestandteil von Schutzkleidung eine Rolle, sondern werden zum Beispiel auch zur Panzerung von Fahrzeugen eingesetzt.

Die Wirkungsweise von weichballistischen Materialien lässt sich folgendermaßen beschreiben: Wenn ein Geschoss auf eine mehrlagige Struktur aus reißfestem weichballistischem Gewebe trifft, dann wird seine kinetische Energie abgebaut, indem das Geschoss beim Durchdringen der einzelnen Gewebelagen diese zerstört oder dehnt. Nach dem Durchdringen mehrerer Lagen ist die Energie vollständig abgegeben und das Geschoss bleibt stecken. Weichballistische Materialien werden hauptsächlich zum Schutz vor Kurzwaffengeschossen oder beschleunigten Metallsplittern eingesetzt.

Schutzsysteme aus weichballistischen Materialien enthalten als wirksame Komponente zumeist Gewebe aus hochfesten synthetischen Fasern auf der Basis aromatischer Polyamide (Aramide). Ein bekannter Handelsname solcher Materialien ist Kevlar oder auch Twaron. Bis heute gibt es keine gesicherten Aussagen darüber, ob und wie sich die Schutzeigenschaften von Aramiden mit zunehmendem Alter verändern. Hersteller von Schutzwesten gewähren derzeitig 10 Jahre Garantie auf die Schutzeigenschaften der Schutzweste - Forderung der Technischen Richtlinie des Polizeitechnischen Instituts (Geßner 2009).

Im Folgenden sollen erste Ergebnisse systematisch durchgeführter Langzeituntersuchungen an AramidGeweben dargestellt werden. Zur Klärung der stofflichen Mechanismen, die einer möglichen altersbedingten Veränderung der Schutzwirkung von Aramiden zugrunde liegen, haben wir sowohl Beschussversuche an polizeilichen Schutzwesten als auch Laborversuche am Aramid-Gewebe durchgeführt.

\section{Stand der Wissenschaft und Technik}

Schon im Jahre 1986 wurde vom amerikanischen »National Bureau of Standards« im Auftrage des »National Institute of Justice« eine Untersuchung zum Langzeitverhalten von ballistischen Schutzgeweben auf der Basis von Kevlar durchgeführt. Die Ergebnisse wurden in dem Bericht »Ballistic Tests of Used Soft Body Armor» zusammengefasst (Frank 1986).

Es wurden jedoch nur wenige Gewebe untersucht (insgesamt 24 Proben unterschiedlichen Alters, unter- 
schiedlicher Herkunft und unterschiedlicher Tragedauer), so dass nur subjektive und allgemeine Aussagen über Trends möglich sind. Zwei Trends werden von den Autoren angegeben:

1. Mit zunehmendem Probenalter kann von den durchgeführten Messungen nicht auf eine Verschlechterung der Schutzeigenschaften geschlossen werden

2. Häufig getragene Schutzwesten scheinen bessere Schutzeigenschaften aufzuweisen als weniger häufig getragene Westen

Im Gegenzug dazu liegen nach einem Bericht der PolizeiFührungsakademie aus dem Jahre 1998 (Polizeitechnisches Institut der Polizei-Führungsakademie 1998) die Grenzwerte V50 (V50 ist die Geschossgeschwindigkeit, bei der die Wahrscheinlichkeit für einen Durchschuss $50 \%$ beträgt) bei gealterten und getragenen Schutzwesten bei drei von vier Schutzwesten-Modellen (verschiedener Hersteller) höher als bei neuen Westen. Über das vierte Westenmodell können laut Bericht aufgrund der wenigen Messergebnisse keine Aussagen gemacht werden. Betrachtet man die Messergebnisse im Einzelnen, stellt man fest, dass die Anzahl der Schutzwesten pro Altersklasse und pro Hersteller in keinem Fall größer als 6 war.

\section{Beschussversuche}

Vorbereitende Arbeiten

Aufgrund einer Kooperation mit dem Zentraldienst der Polizei Brandenburg »ZDPol« standen uns 599 Unterzieh-Schutzwesten zur Verfügung, die anhand unterschiedlicher Merkmale wie Hersteller, Alter, Nutzungsgrad und Produktionscharge kategorisiert wurden. Eine Produktionscharge wird entweder durch die Zertifikatsnummer des Herstellers oder, falls diese nicht bekannt ist, durch eine selbst vergebene Bezeichnung »U2« gekennzeichnet. Der Nutzungsgrad einer Weste wurde per Augenschein geschätzt. Das Ergebnis war eine Datenmatrix über 599 Datensätze, auf deren Grundlage eine Auswahl für die Beschussversuche vorgenommen wurde. Die Kategorisierung ergab 17 verschieden Produktionschargen von fünf verschiedenen Herstellern mit einem Alter zwischen 0 und 18 Jahren.

\section{Versuchsaufbau}

Einige der Beschussversuche wurden im Beschussamt Mellrichstadt, die meisten jedoch auf dem Schießstand des ZDPol durchgeführt. Waffenlauf (siehe Abb. 1) und
Auflage für die Weste sind standardisiert. Die Westenauflage besteht aus einem Block Plastilin-Knete und ähnelt vom Druck- und Formverhalten den Eigenschaften des menschlichen Körpers. Als Geschoss wurde das Projektil $9 \mathrm{~mm}$ Luger verwendet.

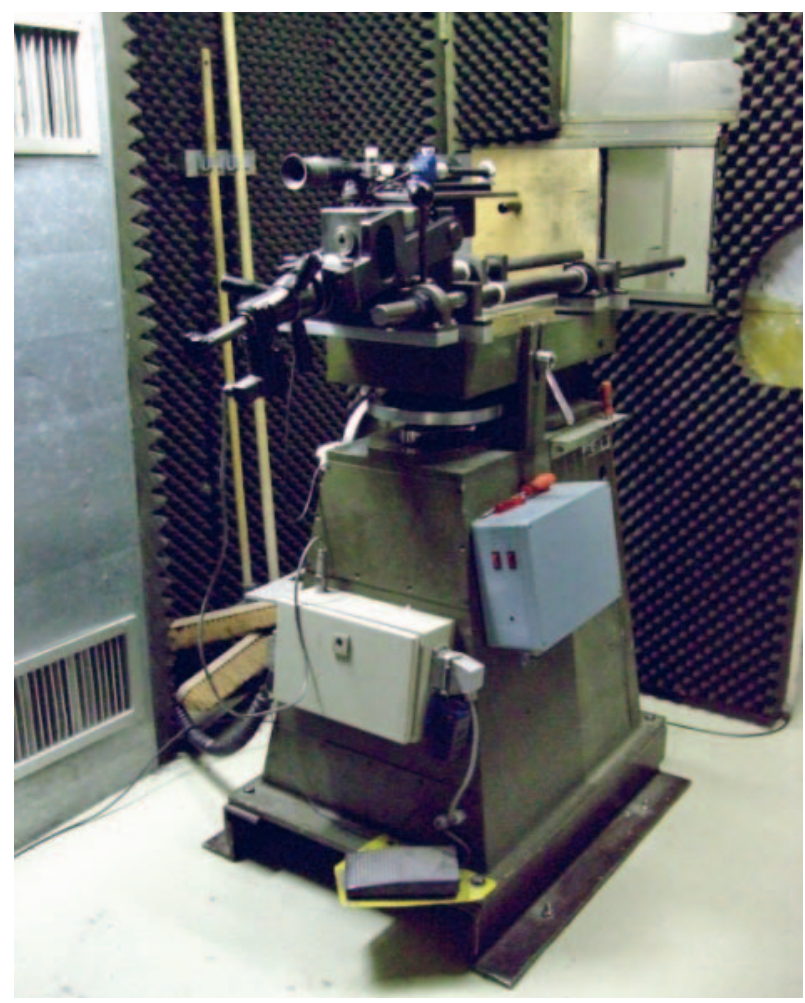

Abb. 1: Beschussvorrichtung in Mellrichstadt; der Waffenlauf zeigt in Richtung des kleinen Fensters

Der Abstand der Weste zum Ausgang des Waffenlaufs betrug $10 \mathrm{~m}$. Ein Hochgeschwindigkeitsmessgerät, welches die Geschossgeschwindigkeit auf eine Millisekunde genau misst, wurde in zwei Meter Entfernung vor dem Westenaufbau platziert. Die Platzierung der Schüsse und ihre Reihenfolge waren durch eine Schablone vorgegeben und wurden durch Kreide oder Wachsstift direkt auf der Weste markiert (siehe Abb. 2).

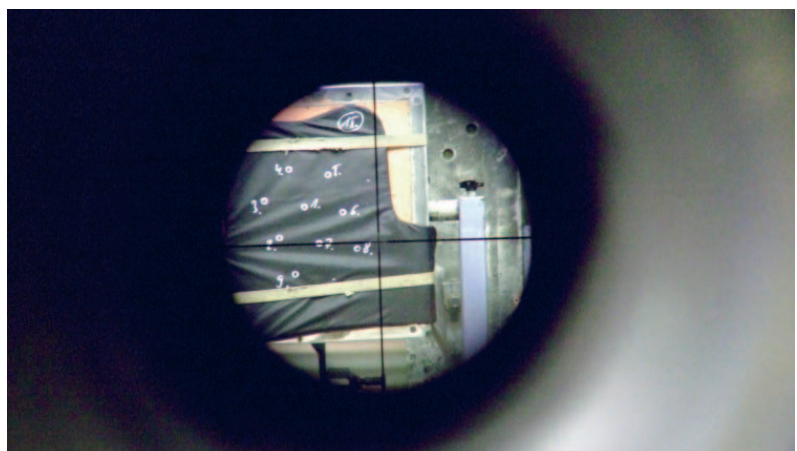

Abb. 2: Blick durch das Zielfernrohr der Beschussapparatur auf die am Plastilin-Block befestigte Schutzweste mit Kreidemarkierungen 
Die Geschosse wurden eigenhändig laboriert, um durch unterschiedliche Pulvermengen die Geschossgeschwindigkeit variieren zu können.

Eine ausführliche Beschreibung des Versuchsaufbaus ist z. B. (Vereinigung der Prüfstellen für angriffshemmende Materialien und Konstruktionen 2009: Seite 1928) zu entnehmen.

\section{Bestimmung der V50-Grenzgeschwindigkeit}

Ein Maß zur Bestimmung der Schutzeigenschaften eines ballistischen Schutzsystems ist die Bestimmung der V50-Grenzgeschwindigkeit. Dieser V50-Wert ist die Geschwindigkeit eines Geschosses, bei der die Wahrscheinlichkeit eines Durchschusses bei 50\% liegt. Zur Berechnung des Wertes gibt es mehrere unterschiedliche Methoden (Kneubuehl 2000: Seite 22-31). Im Rahmen der hier durchgeführten Untersuchungen wurden die Beschusstests nach STANAG 2920 durchgeführt (NATO STANAG 2920 2003). Dabei wurde die Schutzweste an maximal 12 fest definierten Positionen auf der Schutzweste beschossen. Die V50-Grenzgeschwindigkeit berechnete sich aus dem Median von drei Durchund drei Steckschüssen (Kneubuehl 2000: Seite 24), wobei allerdings die Differenz der höchsten und der niedrigsten dieser sechs Geschossgeschwindigkeiten nicht mehr als $30 \mathrm{~m} / \mathrm{s}$ betragen durfte (Polizeitechnisches Institut der Polizei-Führungsakademie 2003: Seite 15).

\section{Streuung der Messwerte}

Bei den Beschussversuchen war festzustellen, dass die V50-Werte auch innerhalb einer Produktionscharge Schwankungen unterliegen, die im Wesentlichen mit dem Verfahren zur Bestimmung der Grenzgeschwindigkeiten und mit Produktionsschwankungen zu tun haben dürften. Ein Beispiel für die Streuung der V50Werte zeigt Abb. 3.

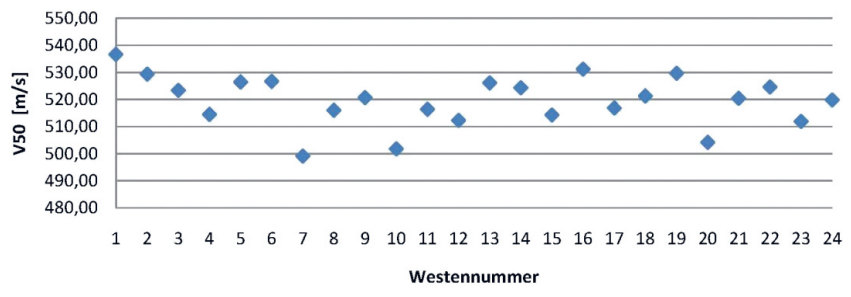

Abb. 3: V50-Werte von 24 verschiedenen 6 Jahre alten Westen der Fa. Mehler aus der Produktionscharge 03Z085-01

Es handelte sich hier ausschließlich um 6 Jahre alte Westen einer Produktionscharge der Fa. Mehler Vario
System GmbH (kurz: Mehler). Die jeweilige V50-Grenzgeschwindigkeit ist durch den blauen Punkt dargestellt.

Die V50-Werte der Westen der Produktionscharge 03Z085-01 lagen in etwa in einem Geschwindigkeitsband von $40 \mathrm{~m} / \mathrm{s}$. Messungen der Streuung an anderen Produktionschargen (auch anderer Hersteller) ergaben sowohl hinsichtlich des Mittelwertes der Versuchsreihe als auch hinsichtlich der Breite des Geschwindigkeitsbandes vergleichbare Ergebnisse.

\section{Gebrauchszustand der Westen}

Um abzuschätzen, in wie weit der Gebrauch einer Schutzweste durch z. B. Walken des Gewebes, Temperaturschwankungen und Kontakt mit Körperschweiß einen Einfluss auf die V50-Grenzgeschwindigkeit hat, wurde im Rahmen der Kategorisierung der Westen der Gebrauchszustand einer Weste per Augenschein geschätzt. Dabei wurde zwischen ungebraucht, gebraucht und stark gebraucht unterschieden. Messungen an über 30 Westen unterschiedlichen Gebrauchszustandes ließen keinerlei Abhängigkeiten des V50-Wertes vom Gebrauchszustand einer Weste feststellen. Bemerkt werden muss an dieser Stelle, dass bei allen derart untersuchten Westen die Schutzhülle gegen UV-Strahlung und Feuchtigkeit, die beide die Schutzeigenschaften von AramidGeweben negativ beeinflussen, unversehrt war.

\section{Langzeituntersuchungen}

Im Folgenden sollen die wesentlichen Ergebnisse der Beschussversuche dargestellt werden, wobei wir uns hier aus Gründen der Übersichtlichkeit nur auf die Darstellung der Ergebnisse, die mit Schutzwesten des Herstellers Mehler gewonnen wurden, beschränken. Weiterhin werden wir an dieser Stelle nur Ergebnisse vorstellen aus Versuchen mit Westen, die nur aus Aramid-Gewebe (evtl. zuzüglich Schockabsorber) bestehen. Westen, die zusätzlich andere Bestandteile wie laminiertes Aramid, Aramid-Gelege oder Stichschutz etc. haben, sind hier nicht einbezogen.

Wir haben bis heute Beschussversuche an Schutzwesten bis zu einem Alter von 18 Jahren durchgeführt. Die Ergebnisse dieser Versuche sind für Westen mit einem Alter von bis zu 10 Jahren durch Laborversuche bestätigt. Laborversuche an Westen, die älter sind als 10 Jahre, laufen momentan. Aus diesem Grunde sollen hier auch nur die Ergebnisse, die für bis zu 10 Jahre alte Westen gewonnen wurden, vorgestellt werden.

Tabelle 1 gibt eine Übersicht über die für die Langzeituntersuchungen herangezogenen Westen der Fa. Meh- 
ler. Die erste Spalte beinhaltet die Chargennummer, die zweite Spalte gibt das Alter der Weste zum Zeitpunkt des Beschusses an, die dritte Spalte gibt das Herstellungsjahr der Weste an und die vierte Spalte zeigt die Anzahl der für die Beschussversuche vorhandenen Westen.

\begin{tabular}{|l|l|l|l|}
\hline $\begin{array}{l}\text { Chargen- } \\
\text { nummer }\end{array}$ & $\begin{array}{l}\text { Alter bei } \\
\text { Beschuss/Jahre }\end{array}$ & $\begin{array}{l}\text { Herstellungs- } \\
\text { jahr }\end{array}$ & Anzahl \\
\hline 01W025A01 & 9 & 2001 & 28 \\
\hline 01W025A01 & 8 & $2001+2002$ & 25 \\
\hline 01W025A01 & 7 & 2002 & 25 \\
\hline 03Z085-01 & 7 & 2003 & 3 \\
\hline 03Z085-01 & 6 & 2003 & 24 \\
\hline 04Z167D01 & 6 & 2004 & 2 \\
\hline 04Z167D01 & 5 & 2004 & 7 \\
\hline U2 & 0 & 2001 & 1 \\
\hline & & & $\Sigma 115$ \\
\hline
\end{tabular}

Tabelle 1: Für die Langzeituntersuchungen beschossene Mehler-Westen aus reinem Kevlar-Gewebe

Abb. 4 zeigt das Ergebnis der Beschussversuche an 115 verschiedenen Westen. Dargestellt ist das arithmetische Mittel aller für ein bestimmtes Alter gemessenen V50Werte als Funktion des Alters. Da bei den Beschussversuchen der Geschossaufbau herstellerbedingt teilweise leicht variierte, sind die V50-Werte diesbezüglich korrigiert worden (Gäbler et al. 2010). Weiterhin brauchte der Ort des Beschusses nicht berücksichtigt zu werden, da Vergleichsmessungen in Mellrichstadt und beim ZDPol in Wünsdorf an Schutzwesten derselben Produktionscharge und desselben Herstellungsjahres keine signifikanten Unterschiede bei den V50-Werten zeigten.

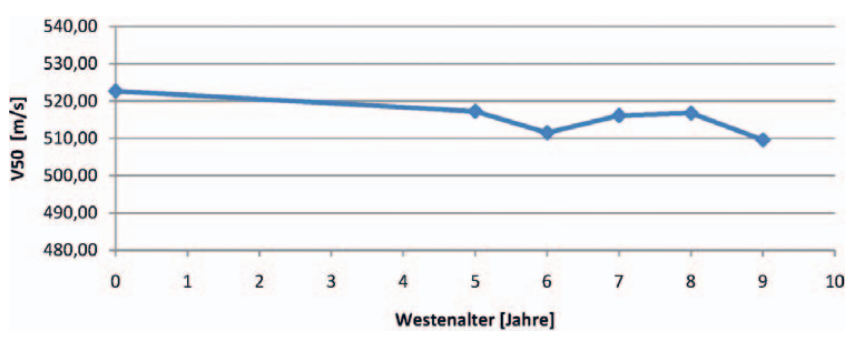

Abb. 4: Arithmetisches Mittel der V50-Werte aller Mehler-Westen gleichen Alters als Funktion des Alters

Westenaufbau und Vergleichbarkeit der Ergebnisse Für unterschiedliche Produktionschargen kann der innere Aufbau einer Weste unterschiedlich sein. Tabelle 2 zeigt den Westenaufbau für die unterschiedlichen Chargen. Mit angegeben ist das Gewicht eines $100 \mathrm{x}$
100 mm großen Flächenstücks einer Lage des AramidGewebes.

\begin{tabular}{|c|c|c|}
\hline $\begin{array}{l}\text { Chargen- } \\
\text { nummer }\end{array}$ & Aufbau & $\begin{array}{l}\text { Flächengewicht } \\
\text { einer Lage } \\
(100 \times 100 \mathrm{~mm}) / \mathrm{g}\end{array}$ \\
\hline 01W025A01 & $\begin{array}{l}32 \text { Lagen Aramid mit einer } \\
\text { X-Naht }(120 \times 120 \mathrm{~mm}) \\
\text { mittig vernäht }\end{array}$ & 2,1 \\
\hline 03Z085-01 & $\begin{array}{l}32 \text { Lagen Aramid mit einer } \\
\text { X-Naht }(120 \times 120 \mathrm{~mm}) \\
\text { mittig vernäht und in Folie } \\
\text { eingeschweißt }\end{array}$ & 2,1 \\
\hline 04Z167D01 & $\begin{array}{l}32 \text { Lagen Aramid mit einer } \\
\text { X-Naht }(120 \times 120 \mathrm{~mm}) \\
\text { mittig vernäht und in Folie } \\
\text { eingeschweißt }\end{array}$ & 2,1 \\
\hline U2 & $\begin{array}{l}32 \text { Lagen Aramidgewebe } \\
\text { mit X-Naht }(100 \times 100 \mathrm{~mm}) \\
\text { und mit zwei senkrechten } \\
\text { Nähten }(70 \mathrm{~mm}) \text { am oberen } \\
\text { und unteren Rand vernäht }\end{array}$ & 2,3 \\
\hline
\end{tabular}

Tabelle 2: Westenaufbau und Flächengewicht für unterschiedliche Produktionschargen

Die Westen der Chargen 01W025A01, 03Z085-01 und 04Z167D01 sind vom Aufbau her identisch und haben auch das gleiche Flächengewicht bezogen auf eine Stofflage. Die Westen der Charge U2 unterscheidet sich vom prinzipiellen Aufbau her nicht von den Westen der anderen drei Chargen. Die Weste der Charge U2 hat bei ebenfalls 32 Stofflagen ein leicht höheres Flächengewicht verglichen mit den Chargen 01W025A01, 03Z085-01 und 04Z167D01.

Der V50-Wert für die Charge U2 liegt in der gleichen Größenordnung wie der V50-Wert für die Chargen 01W025A01, 03Z085-01 und 04Z167D01 (vgl. Abb. 5), obwohl in dem Fall (U2) das Flächengewicht etwas größer ist. Eine mögliche Erklärung für dieses Verhalten könnte sein, dass sich über die Jahre im Herstellungsprozess des Gewebes etwas geändert hat.

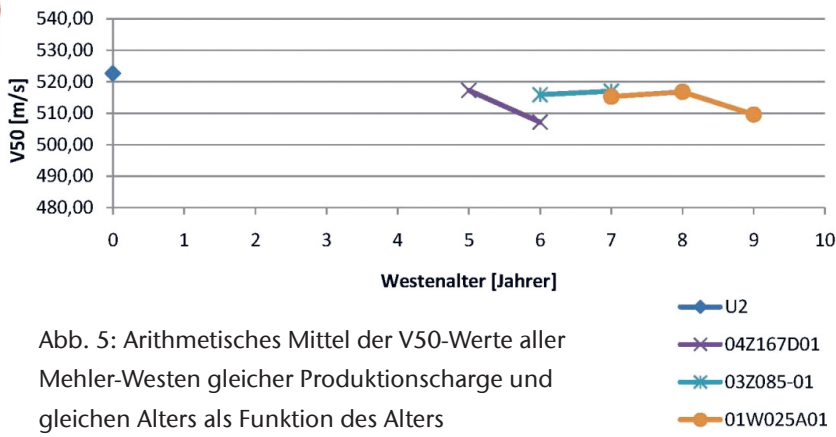


Um festzustellen, welchen Einfluss eine zusätzliche Stofflage auf den V50-Wert der Weste haben würde, hatten wir unterschiedliche Westen einer Produktionscharge (15 Jahre alt) beschossen und dabei die Anzahl der Stofflagen von Weste zu Weste reduziert. Abb. 6 zeigt das Ergebnis dieser Versuche.

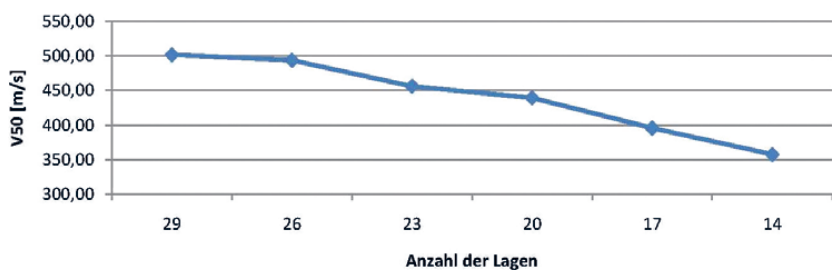

Abb. 6: V50-Werte einzelner Westen einer Produktionscharge als Funktion der Lagenzahl

Der Abfall der V50-Grenzgeschwindigkeit mit verringerter Lagenzahl ist nahezu linear und beträgt hier etwa $9,5 \mathrm{~m} / \mathrm{s}$ pro Lage.

\section{Bewertung der Beschussversuche}

Den Ergebnissen der von uns durchgeführten Beschussversuche an polizeilichen Unterzieh-Schutzwesten lässt sich bis zu einem Westenalter von 10 Jahren weder eine altersbedingte Verschlechterung noch eine altersbedingte Verbesserung der Schutzeigenschaften entnehmen. Der in Abb. 4 erkennbare leichte Abfall des V50Wertes liegt deutlich innerhalb des Streubandes (vgl. Abb. 3) und ist daher nicht signifikant. Erste Versuche an noch älteren Westen zeigen ebenfalls keine altersbedingten Änderungen der Schutzeigenschaften.

\section{Laboruntersuchungen}

Zusätzlich zu den Beschussversuchen an Schutzwesten wurden Zugversuche an Aramid-Gewebestreifen durchgeführt. An dieser Stelle sollen nur grob der Versuchsaufbau sowie die wesentlichen Ergebnisse dargestellt werden. Eine ausführlichere Beschreibung der Zugversuche sowie der ebenfalls durchgeführten DSC-Messungen an Kevlar sind (Beckmann et al. 2010) zu entnehmen.

Die Zugversuche wurden mittels der Universalprüfmaschine Zwick Z20 durchgeführt. Dazu wurden aus vorher beschossenen Westen Gewebestreifen von ca. $3 \mathrm{~cm}$ Breite und mit maximaler Länge ausgeschnitten (Areale mit Schädigungen durch Beschuss wurden nicht verwendet) und auf $2 \mathrm{~cm}$ ausgefranst, d.h. die quer zur Zugrichtung liegenden Faserbündel (Rovings) wurden an den Ecken frei gelegt.
Tabelle 3 gibt eine Übersicht derjenigen Westen der Fa. Mehler, aus denen Gewebestreifen entnommen und untersucht wurden.

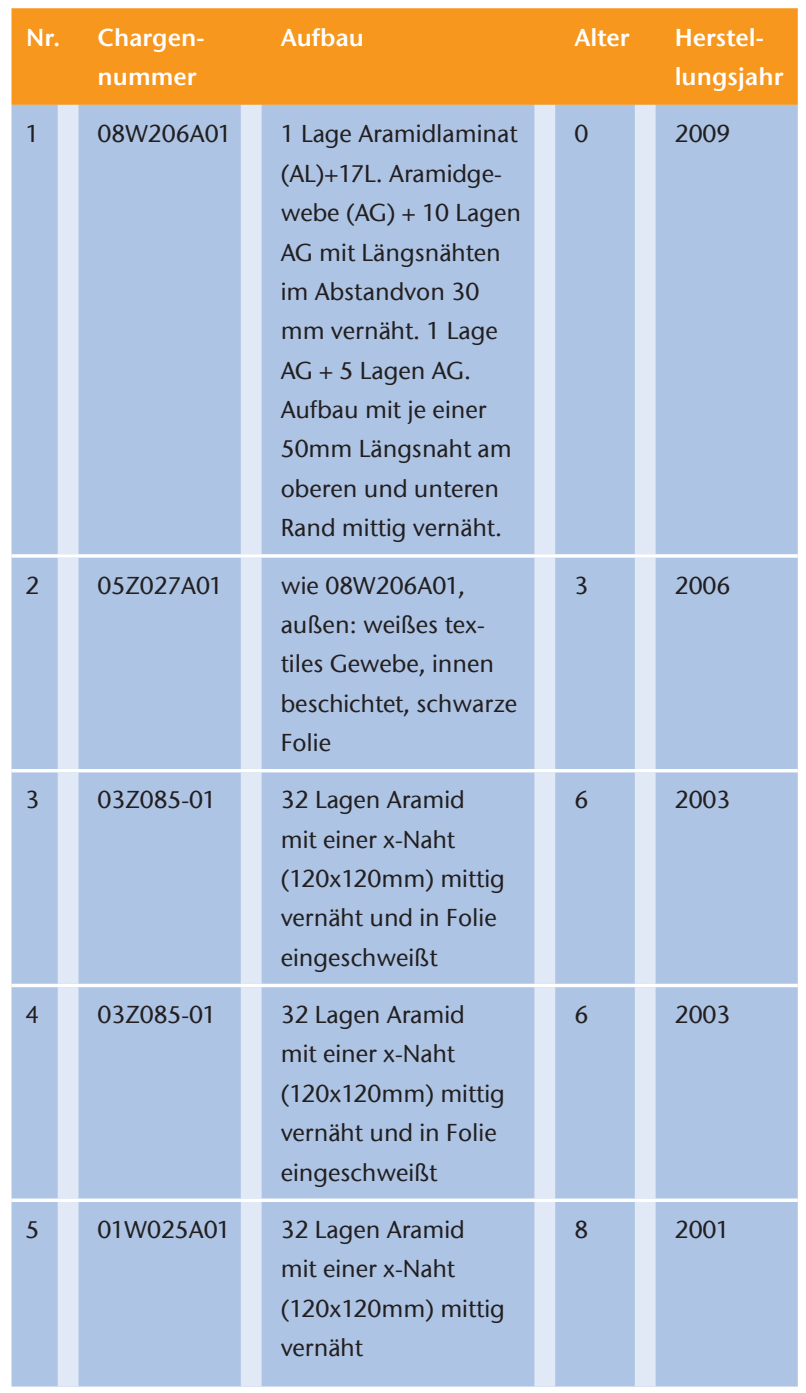

Tabelle 3: Für die Zugversuche benutzte Mehler-Westen

Abb. 7 zeigt die Zugfestigkeit als Funktion des Gewebealters, während Abb. 8 die dazugehörigen Zerstörungsenergien darstellt.

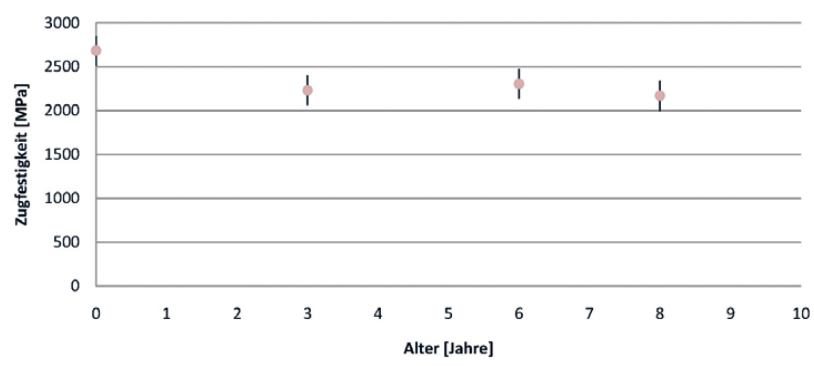

Abb. 7: Zugfestigkeit von Kevlar-Gewebe einschließlich Vertrauensintervall als Funktion des Gewebealters 


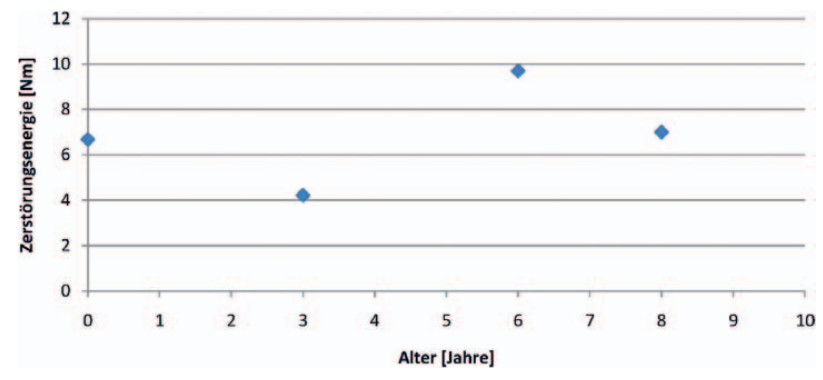

Abb. 8: Zerstörungsenergie zu Abb. 7

Die Zugversuche am Aramid-Gewebe bestätigen die Ergebnisse der Beschussversuche.

\section{Ausblick}

In der nächsten Zeit sollen folgende weitere Untersuchungen durchgeführt werden:

- Verifikation der Beschussversuche an bis zu 18 Jahre alten Westen durch Messungen im Labor

- Ausweitung sowohl der Beschussversuche als auch der Labormessungen an bis zu 25 Jahre alten AramidGeweben

- Gezielte und verifizierbare Untersuchungen in der Klimakammer zum Einfluss von UV-Strahlung und Luftfeuchtigkeit auf die Schutzeigenschaften von Aramiden

\section{Danksagung}

Dieses Projekt wird vom Bundesministerium für Wirtschaft und Technologie (BMWI) finanziell unterstützt.

\section{Literatur}

Geßner, E. (2009): Langzeitverhalten von ballistischen Schutzausrüstungen, Vortrag im Rahmen der Fachkonferenz der Polizei »Waffen- und Gerätewesen«.

Frank, D. E. (1986): Ballistic Tests of Used Soft Body Armor; U.S. Department of Commerce, National Bureau of Standards.

Polizeitechnisches Institut der Polizei-Führungsakademie (1998): Langzeitverhalten von Schutzwesten IV.

Vereinigung der Prüfstellen für angriffshemmende Materialien und Konstruktionen 2009: VPAM BSW 2006, Prüfrichtlinie »Ballistische Schutzwesten «

Kneubuehl, B. P. (2000): Ballistischer Schutz, Thun.

NATO STANAG 2920 (2003): Ballistic Test Method for Personal Armour Materials and Combat Clothing.

Polizeitechnisches Institut der Polizei-Führungsakademie (2003): Technische Richtlinie »Ballistische Schutzwesten «.

Gäbler, A, Hendrix, M., Geßner, E. (2010): Einfluss von Änderungen der Prüfmunition hinsichtlich der Vergleichbarkeit von Ergebnissen bei der Ermittlung von ballistischen Grenzwerten nach STANAG 2920, pVt Polizei Verkehr Technik (in Druck).

Beckmann, S., Herzog, M., Hendrix, M. (2010): Untersuchungen zur Langzeitstabilität von ballistischen Schutzwesten, GIT Sicherheit + Management, GIT Verlag, Darmstadt (in Druck)

\section{Autoren}

Dipl.-Wirt.-Inf. (FH) Anja Gäbler

Technische Hochschule Wildau [FH]

anja.gaebler@th-wildau.de

\section{Prof. Dr. Michael Hendrix}

Technische Hochschule Wildau [FH]

michael.hendrix@th-wildau.de

\section{Dr. Michael Herzog}

Technische Hochschule Wildau [FH] michael.herzog@th-wildau.de

\section{Eberhard Geßner}

Leiter Produkt-Kunden-Management Technik - ZBTL2

Zentraldienst der Polizei Brandenburg

Am Baruther Tor 20, 15806 Zossen

eberhard.gessner@polizei.brandenburg.de 